\title{
Recent Advances in IR Laser Chemistry
}

\author{
A. KALDOR, R. L. WOODIN and R. B. HALL
}

Corporate Research-Science Laboratories, Exxon Research and Engineering Company, P.O. Box 45, Linden, New Jersey 07036

The selective absorption of IR laser radiation by specific molecules in complex mixtures or by specific functional groups (or chromophores) in a complex molecule offers an attractive way to energize molecules and influence the path and progress of chemical reactions. Unimolecular decomposition of a selectively excited isotopic species by IR multiple-photon processes has been demonstrated for a very large number of molecules bearing a broad range of elements, but the early promise of the field to do "bond selective" chemistry by localization of energy within a molecule has been frustratingly elusive to achieve. The prevailing view in the laser chemistry community is that due to the fundamental nature of incoherent infrared multiple-photon excitation the search for "bond selective" chemistry via this route is comparable to the search for fool's gold. Many would suggest that other modes of vibrational energy deposition, such as direct excitation of high overtones, offer more promising paths toward success. The fact that classical trajectory studies show that in many reactions the potential surface leading to the formation of a specific reaction product involves at any one time only a limited subset of phase space will continue to challenge chemists in their search for new and novel methods to drive chemical reactions. We belong to that subset of frustrated chemists who believe that the time scale of most experiments performed to date as well as the molecules selected to test these ideas have by their very nature, at the outset precluded the likelihood of success. In this report we describe experiments which probe several key elements of a hypothetical "mode 
selective" process which need to be understood and taken into account in the design of the kind of experiment which may lead to the demonstration of energy localization and novel chemistry. Even if the final objective is not attainable we can and do learn about aspects of molecular spectroscopy, energetics, and dynamics which are fundamental to many facets of chemical physics. These experiments probe $3 \mu \mathrm{m}$ IR multiple-photon absorption, direct and sensitized laser excitation pyrolysis, and surface laser chemistry-work in these three areas point toward specific directions for achieving the ultimate objective.

One question we want to address is whether or not the structure and dynamics of highly vibrationally excited molecules is conducive to localized excitation and subsequent localized chemistry. Recent results from several laboratories using two IR lasers simultaneously exciting and driving a molecule to dissociation show that, even in the dense vibrational quasicontinuum, structure and particular states of the molecule enhance multiple-photon excitation process. We have measured energy deposition due to $3 \mu \mathrm{m}$ infrared laser multiplephoton excitation of propylene and deuterated propylenes using optoacoustic detection. The data show that multiple-photon excitation occurs most readily on the methyl group of propylene, and that efficient excitation at the methyl group requires the $\mathrm{CH}$ group on the adjacent carbon. As a result, multiple-photon excitation of propylene involves energy deposition directly into several molecular $\mathrm{CH}$ sites. This suggests that from the outset, irrespective of intramolecular energy relaxation, that the objective of spatially localized "mode selective" excitation by IR multiple-photon excitation cannot be achieved. But our results also offer a hint that all is not lost. The data indicate that the $\mathrm{CH}_{2}$ group furthest from the $\mathrm{CH}_{3}$ group participates less than the $\mathrm{CH}$ group on the carbon next to the $\mathrm{CH}_{3}$ group. Intermode coupling is thus seen to decrease with spatial separation of the vibrational modes leaving open the possibility of "region specific" intramolecular excitation. It may be possible to design molecules with appropriate vibrational modes so as to create efficient chromophores for multiple-photon excitation and at the same time incorporate heavy mass constituents to block energy flow from one point of the molecule to another in order to achieve selective reaction.

The second issue we have probed is the chemistry one can observe depending on the temperature profiling of the reaction system. It is 
essential to understand the unusual product distributions one observes from direct vibrational excitation and how they differ from those due to homogeneous heating or from heterogeneous (wall or surface) effects. Recently we have examined the IR multiple-photon driven laser chemistry of $\mathrm{C}_{2} \mathrm{H}_{6}$ using $3 \mu \mathrm{m} \mathrm{CH}$ mode excitation, and $\mathrm{SF}_{6}$ sensitized homogeneous laser pyrolysis using two different $\mathrm{CO}_{2}$ laser pulse shapes. Our results are quite interesting and have important implications for dealing with experimental results in IR laser chemistry.

The $3 \mu \mathrm{m}$ direct excitation produces reactive intermediates in a "cold" bath gas. Thus chain propagation and other secondary reactions with high activation energy are closed off. The direct excitation also produces high energy radicals, e.g., $\mathrm{CH}_{2}$ :, in the primary step. Collisional quenching is observed to inhibit high energy radical reactions. The products of this reaction are mostly methane, with a very low yield of ethylene and somewhat higher yield of $\mathrm{C}_{3} \mathrm{H}_{8}$ and $\mathrm{C}_{3} \mathrm{H}_{4}$.

Indirect sensitized laser pyrolysis produces somewhat lower excitation levels, producing primary radicals via the lowest energy pathway in a "hot" bath gas. Collisional quenching enhances chain termination and the evolution of the temperature profiles can strongly affect product distributions. In this case ethane yields much lower $\mathrm{CH}_{4}$ products, and the chain propagation produces mostly $\mathrm{C}_{2} \mathrm{H}_{4}$ with only trace $\mathrm{C}_{3}$ and $\mathrm{C}_{4}$ chain termination products. Kinetic steering and fine tuning of the product distribution can be achieved by varying the pulse shape of the energy deposition system; it is possible to reduce the $\mathrm{C}_{2} \mathrm{H}_{4}$ yield by a factor of 2 and increase the $\mathrm{C}_{4}$ yield to about $60 \%$ by extending by an order of magnitude the long, low energy tail of the $\mathrm{CO}_{2}$ laser used to couple energy into the system. These results are important when one considers the design of experiments to test energy localized chemistry in molecules. Rapid local excitation may yield primary chemical products whose ultimate reaction path may be profoundly influenced by the energy decay of the system. Molecules potentially can be designed with chromophores which may simplify the reaction progress, as described previously. Rapid quenching of the reaction system is one way to simplify the problem-this means localized heat sinks are required. Heavy atom molecular constituents may block energy flow and also act as local heat sinks. One promising area to explore which may provide the necessary clues is that of laser induced reactions of surface adsorbed molecules. 
The first step here is the examination of laser heating of a surface and measurement of laser desorbed species for the case of a molecule known to undergo dissociation in competition with desorption. We picked methanol on NI as one example. The results are interesting. We find that when $\mathrm{CH}_{3} \mathrm{OH}$ is adsorbed at about $90 \mathrm{~K}$, the molecular entity is desorbed intact by rapid laser induced surface heating $\left(10^{11} \mathrm{Ks}^{-1}\right)$; only a very small amount of $\mathrm{H}_{2}$ and $\mathrm{CO}$ is produced from the decomposition pathways. The amount of $\mathrm{CH}_{3} \mathrm{OH}$ desorbed intact decreases montonically as the nickel is heated to about $180 \mathrm{~K}$, until all is gone at that temperature. Beyond that point to about $230 \mathrm{~K}, \mathrm{H}_{2}$ and $\mathrm{CO}$ both continue to build up on the surface. The $\mathrm{H}_{2}$ yield appears to plateau at about $260 \mathrm{~K}$, while $\mathrm{CO}$ build up continues till the maximum is reached at about $300 \mathrm{~K}$. These results suggest that selective bond breaking might be achieved if the bond is weak enough and the heating is rapid enough. The first step in the dissociation of $\mathrm{CH}_{3} \mathrm{OH}$ on $\mathrm{Ni}$ is:

$$
\left.\mathrm{CH}_{3} \mathrm{OH}_{(\mathrm{ads}}\right) \longrightarrow \mathrm{CH}_{3} \mathrm{O}_{(\mathrm{ads})}+\mathrm{H}_{(\mathrm{ads})}
$$

We have learned that the methoxy radical is bound to the surface more strongly than $\mathrm{CO}$. In fact a gradual stripping of $\mathrm{H}$ atoms is preferred to the desorption of the molecular fragment. These results are tentative, and will require much more detailed studies to prove conclusively. But, they do suggest that the surface heat sink idea for localized IR laser chemistry has potential.

In summary, we suggest that the design of localized "bond selective" vibrationally excited and initiated reactions requires an in-depth understanding of the energy deposition mechanism, and that the insights obtained so far steer the research in the specific directions discussed above. We believe that the excitation or energy deposition should preferably be done via a $\mathrm{CH}$ stretch (or other localized, high frequency absorption feature), and that it be done in $10^{-10}$ seconds or less. Secondly, we suggest that heavy atom constituents be built into the molecular framework to block energy flow and that the thermal and direct excitation laser chemistry be well understood. We suggest that surfaces or clusters may offer attractive substrates as energy sinks and blocking agents. Finally, closer interaction between theory and experiments is essential for the design and analysis required to find the path and convincingly demonstrate that the elusive "mode selective" or "localized" chemistry is possible. 